\title{
Representation of Happiness in Russian and American Culture (Based on a Student Sample)
}

\section{Svetlana Yu. Zhdanova, Anna V. Pecherkina}

Department of Developmental Psychology, Perm State National Research University, Russian Federation

\section{Introduction}

The category of happiness in modern psychological literature is often synonymous with categories of subjective well-being and life satisfaction. However, such an actual identification leads to the fact that the level characteristics of general life satisfaction and the determinants of happiness are studied, while the description of happiness itself as a state experienced in time is leveled.

In the framework of current study, happiness is viewed as a "temporary" state of a person, in contrast to a "stable" state, which in its content appears as an equivalent of the concept of "life satisfaction". This approach allows us to determine the subjective characteristics that reflect the features of experiencing the happiness' state in Russian and American culture, that determines the novelty of the present study

\section{Purpose of the study}

The purpose of the study was to identify the characteristics of the happiness' state representation in Russian and American cultures. The main hypothesis of the study was the assumption that in verbal representation of the state of happiness, it is possible to distinguish general and specific tendencies in connection with cultural belonging.

\section{Methods}

The study involved 76 Russian students from Perm State National Research University (46 female, 30 males; average age $-20,3$ years) and 50 American students from Lyndon State College (Vermont, USA) and Delta State University (Mississippi, USA) (22 female, 28 males, average age $-20,4$ years).

> A narrative interview was used as the main method of data collection. Respondents were asked to recall the situation when they felt happy and talk about it. Further recordings were analyzed.

\section{$>$ The study received 126 videotapes, representing a free narration about events related to experiencing a state of happiness. A scrip was compiled for each video interview (interviews in English language were translated into Russian). \\ $>$ To identify significant differences and conduct a comparative analysis of the frequency of verbal categories occurrence in the representation of happiness' state among Russian and American respondents, the Fisher $\varphi$-angle transform criterion was used

Results

\section{Subcategories of content analysis describing the reasons of} happiness in American and Russian cultures American sample

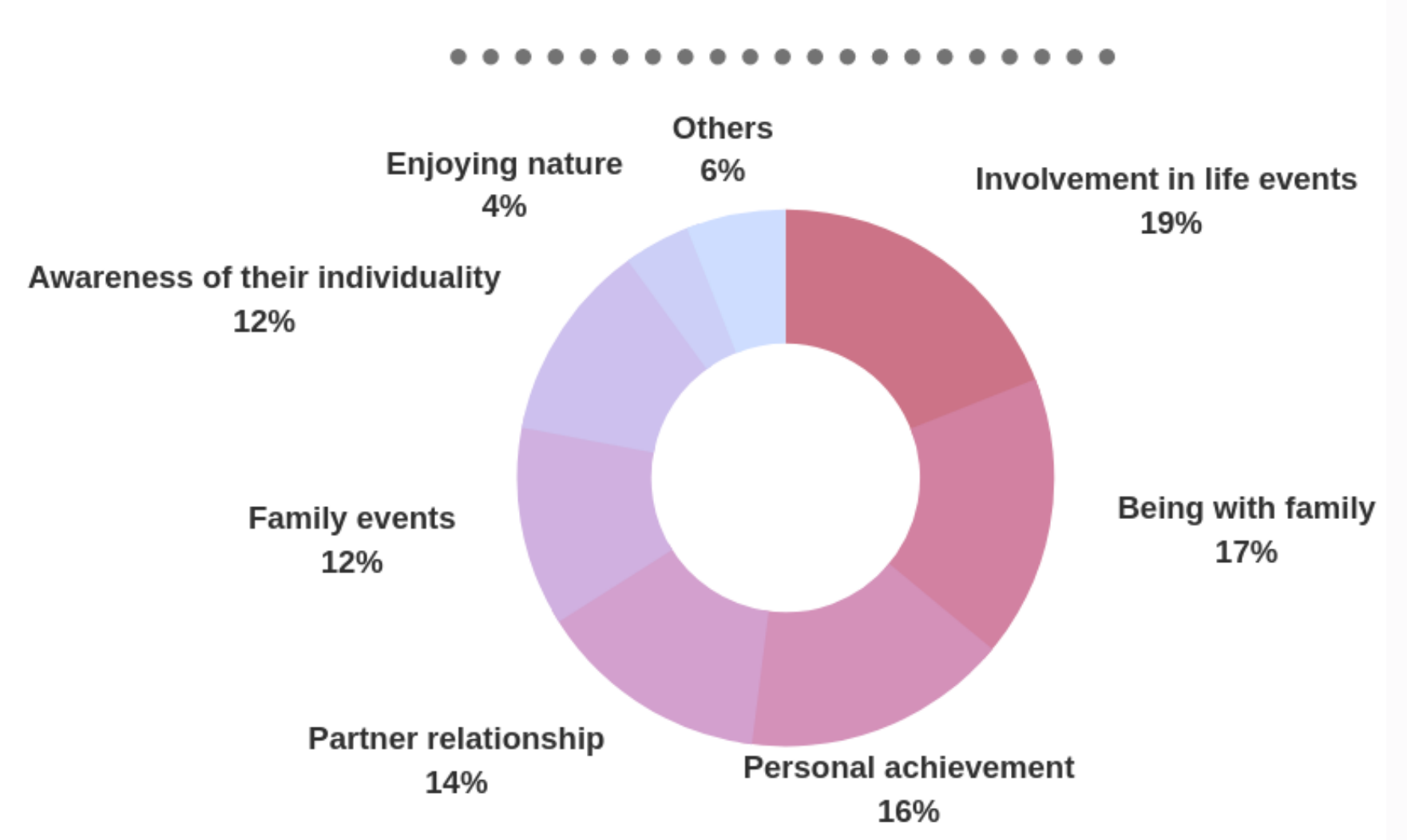

In American narrative, happiness is defined as a state of involvement in life events; reflecting the peculiarities of relations with the family, with the opposite sex; dedication for any business. Happiness in the representation of American respondents appears as a condition due to procedural characteristics. A variable feature that manifested in the American sample is the description of the external expressive characteristics of happiness' state - the description of smiles, laughter, phrases that accompany this state, referring to categories of physical warmth. Based on the obtained results it can be concluded that American respondents are more likely to appeal to the object description of the external expressive characteristics in representation of happiness.

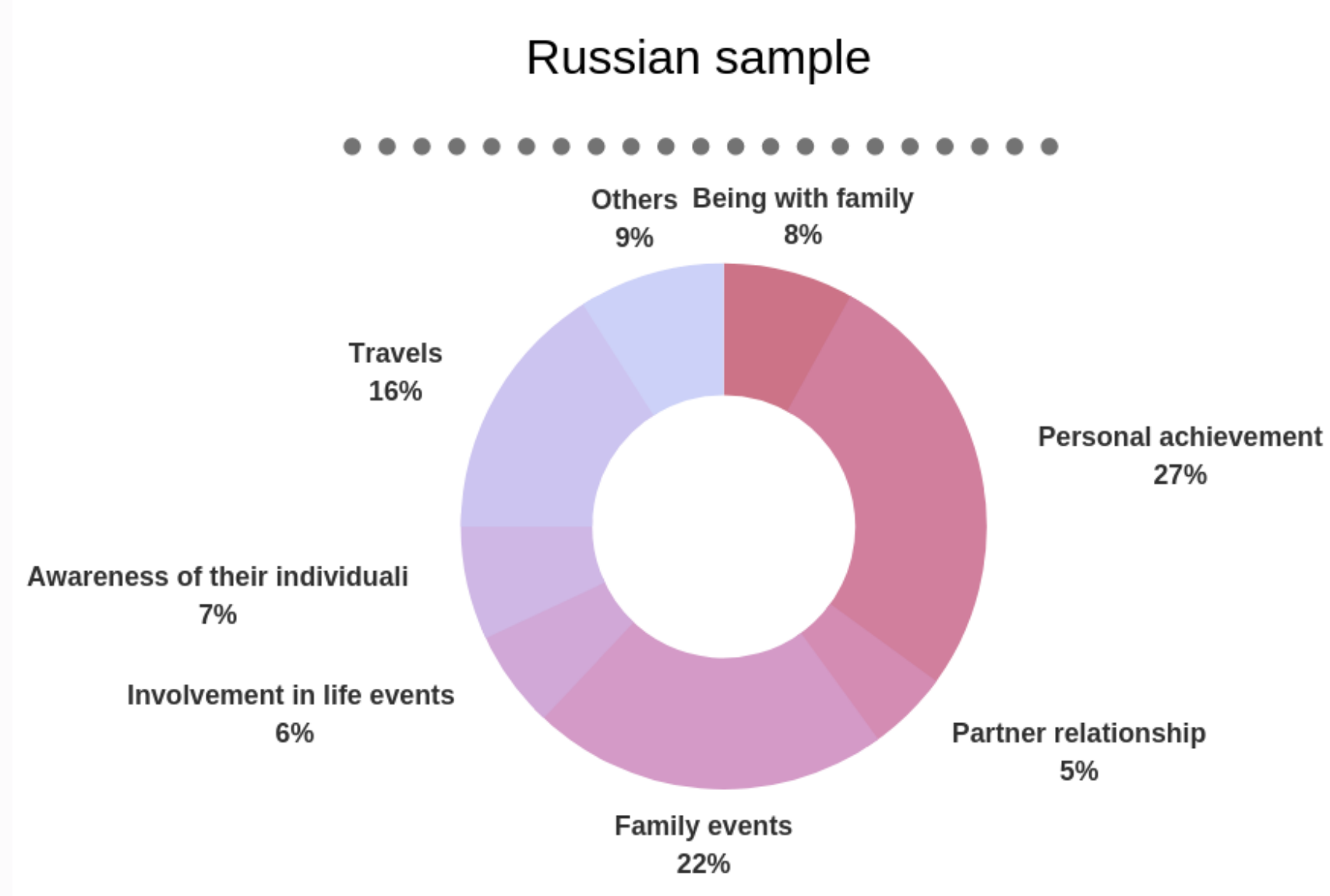

The variability at verbal level of representation among Russian students manifests in referring to the categories describing the duration and variability of happiness' state, a reflection of its atypical in terms of representation in the experience of the subject. Russian respondents more often turn to the description of happiness from the position of internal experiences, based on reflection, while this description is developed and affects changes in the cognitive sphere, reflects the phenomena of the motivational sphere - desire, expectation.

\section{Conclusions}

There is a general trend in the representation of happiness' state at verbal level that is connected with regularity in addressing strategies for describing the state. Thus, the prevailing strategy is the position of subject description. From this descriptive point respondents more often pay attention to the causes of the emotional state, characteristics of internal experiences that accompany this state. Such result correlates with the theoretical propositions that the main characteristic of the mental state image is focusing on internal experiences. From the inner experiences of the state position, the attention of the subject shifts to the description of attitudes towards events and situations, their expression through metaphorical images. This indicates an appeal to the object strategy, an external observer, in which the state is reflected in connection with the objects of the external world.

www.psu.ru 